Classification

Physics Abstracts

$75.30 \mathrm{C}-75.50 \mathrm{~K}-75.60 \mathrm{~J}$

\title{
High field magnetization of two types of shortly correlated systems : random anisotropy systems and nanoparticles
}

\author{
B. Barbara ( $\left.{ }^{1}\right)$, J. Filippi ( $\left.{ }^{1}\right)$, A. Marchand ( $\left.{ }^{1}\right)$, P. Mollard ( $\left.{ }^{1}\right)$, V. S. Amaral ( $\left.{ }^{2}\right)$, \\ X. Devaux $\left({ }^{3}\right)$ and A. Rousset $\left({ }^{3}\right)$ \\ (1) Laboratoire de Magnétisme Louis Néel, CNRS, 38042 Grenoble Cedex, France \\ ( ${ }^{2}$ Centro de Fisica, Universidade do Porto INIC, 4000 Porto, Portugal \\ $\left({ }^{3}\right)$ Laboratoire de Chimie des matériaux inorganiques $\left({ }^{*}\right)$, Univ. Paul Sabatier, 31062 Toulouse \\ Cedex, France
}

(Received 15 May 1991, accepted in final form 18 September 1991)

\begin{abstract}
The magnetization curves of two types of systems (random anisotropy amorphous alloys and ferromagnetic nanoparticles) having finite correlation lengths have been measured and analysed in a wide range of magnetic fields. The results show how these measurements allow us to determine i) the ferromagnetic (thermal) and the atomic (quenched) correlations in amorphous alloys and ii) the quenched distribution of nanoparticles and their mean magnetic moments (role of chemical disorder at the interface boundaries with the matrix). A general discussion involving both types of systems will conclude this paper.
\end{abstract}

\section{Introduction.}

The application of a magnetic field on ordered or disordered systems with ferromagnetic interactions always reduces transverse components of the magnetization and also transverse correlation lengths. The correlative increase of the measured magnetization $M(H)$ is therefore directly related to the $\xi(H)$ variation. In ferromagnets, where long range ferromagnetic order takes place below the Curie temperature, $\xi(0)$ is infinite for a magnetization fraction which increases when the temperature decreases. In disordered systems $\xi(0)$ is truncated to a limit $\xi_{1}(0)$ depending on the nature of the disorder. In random anisotropy systems with ferromagnetic interactions $\xi_{1}(0) \approx R_{\mathrm{a}}\left(\frac{J}{D}\right)^{2}$ where $R_{\mathrm{a}}$ is the distance of correlation of anisotropy directions and $J / D$ the local exchange to anisotropy energy $[1,2]$. In non interacting small particles $\xi_{1}(0)$ is simply equal to $R_{\mathrm{D}}$ the particle size. It is obvious that in both systems the form $\xi(H)$ must depend sensitively on the cut-off value $\xi_{1}(0)$, especially in low fields. The laws of approach to saturation $M(H)=M(\infty)-$ $\Delta M(H)$ must therefore depend on the ratio $J / D$ in RASFI and on the particle sizes $R_{\mathrm{D}}$ in NISP.

$\left.{ }^{*}\right)$ URA-CNRS 1311. 
In this paper, we would like to show that it is now possible to obtain very good fits of the magnetization curves of amorphous alloys at zero Kelvin i.e. to understand in detail and quantitatively their low temperature magnetic properties [2-5]. Concerning NISP we show that a very simple dynamical scaling model allows us to fit the magnetization curves over a wide range of fields and temperatures [6]. This scaling plot allows us to determine the particles size distributions and their mean magnetic moment.

\section{Approach to saturation in random anisotropy systems with ferromagnetic interactions.}

High field magnetization measurements have been performed on the series of amorphous alloys $\mathrm{Dy}_{x} \mathrm{Gd}_{1-x} \mathrm{Ni}$ at the Service National des Champs Intenses, Grenoble, between 1.5 and $4.2 \mathrm{~K}$ up to magnetic fields of $15 \mathrm{~T}$. In order to compare our results to the theory which is safety valid only at zero Kelvin we have performed our experiments at temperatures sufficiently low so that $M(H, T)$ was, within the accuracy of our experiments, independent of the temperature.

The interesting points concerning the series of amorphous alloys $\mathrm{Dy}_{x} \mathrm{Gd}_{1-{ }_{x}} \mathrm{Ni}$ are the following :

i) the nickel with a filled band is non magnetic ;

ii) the ratio, $D / J$ of the local anisotropy to exchange energy can be systematically changed from 1 in DyNi to about 0.01 in GdNi [7].

Raw magnetization results are given in figure 1. Qualitatively they show that saturation becomes more difficult with increasing $D / J$ ratio (i.e. $x$ ). The Pauli paramagnetic susceptibility has been estimated from the high temperature susceptibility of the form $\chi=\chi_{0}+C /(T-\theta)$. Within error bars we always found the same value $\chi_{0}=(8 \pm 1) \times 10^{-4} \mu_{\mathrm{B}} / \mathrm{kOe}$. When comparing the experimental data to theoretical results it is important to take into account the Pauli contribution to the magnetization especially in low anisotropy systems where $\chi_{0} H$ is normally of the same order as $\Delta M=M_{\mathrm{s}}-M_{\text {obs }}(H)$ (where $M_{\mathrm{s}}$ represents the saturation magnetization and $M_{\text {obs }}$ the observed one).

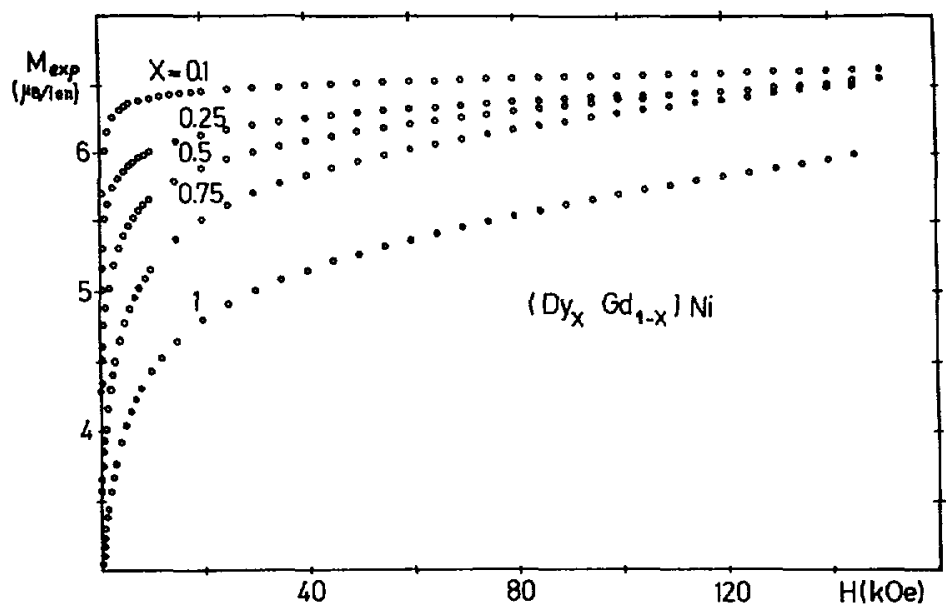

Fig. 1. - Raw magnetization curves measured at $4.2 \mathrm{~K}$ on different samples of the series $\mathrm{a}-\mathrm{Dy}_{x} \mathrm{Gd}_{1-x} \mathrm{Ni}$. 
In this paper we shall restrict our analysis to the case of the system a-Dy ${ }_{0.1} \mathrm{Gd}_{0.9} \mathrm{Ni}$ in which the ratio $D / J$ is of the order of 0.1 , that is small enough to satisfy the assumption of the theory of Chudnovsky et al. [1-5] according which $D / J \ll 1$. Figure 2 shows an excellent fit [5] of the magnetization curve of this sample between $2 \mathrm{kOe}$ and the highest measuring field of $150 \mathrm{kOe}$ to the formula [2] :

$$
M=M_{\mathrm{s}}+\chi_{0} H-\frac{A^{2}}{15 p(1+p)^{3}}
$$

where

$$
\Lambda=K R_{\mathrm{a}}^{2} \sqrt{M_{\mathrm{s}} / A}, \quad K=D / a^{3}, \quad A=z J / 12 a,
$$

$p=\sqrt{\left(H+H_{\mathrm{c}}\right) / H_{\mathrm{co}}}, H_{\mathrm{co}}=2 A / M_{\mathrm{s}} R_{\mathrm{a}}^{2} \cdot R_{\mathrm{a}}$ is the characteristic correlation length of anisotropy directions and $a$ is the mean distance between rare-earth atoms ( $z$ nearest neighbours). $H_{\mathrm{c}}$ is the uniform anisotropy field. This formula which includes both limiting field regimes, was obtained using an exponential correlation function of anisotropy directions $C(r)=$ $\exp \left(-r / R_{\mathrm{a}}\right)$.

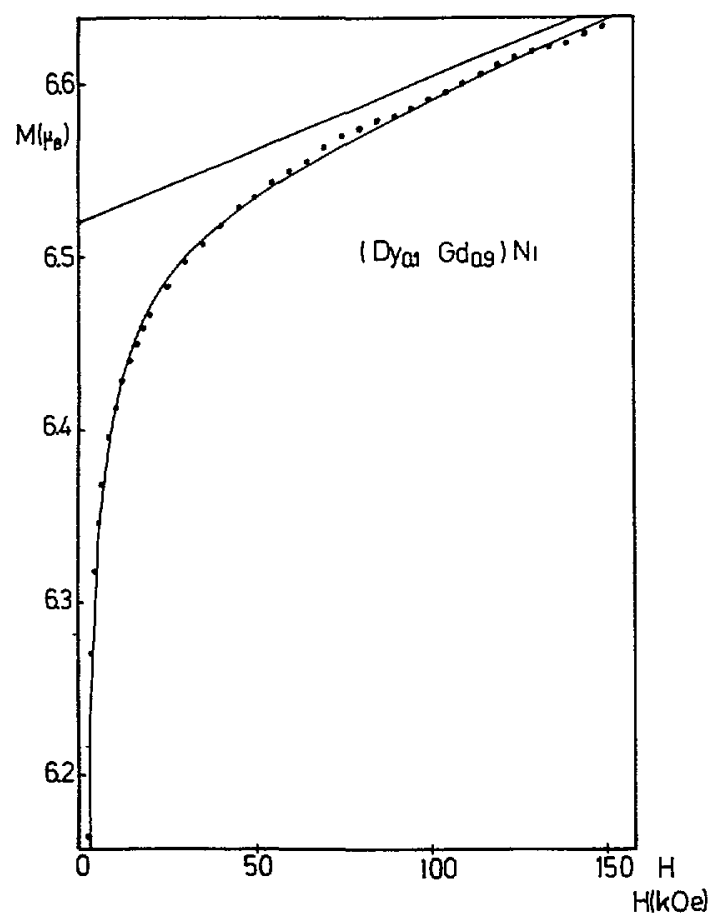

Fig. 2. - Measured magnetization of a-Dy ${ }_{0.1} \mathrm{Gd}_{0.9} \mathrm{Ni}$ (data points). The straight line represents the Pauli paramagnetic susceptibility $M=\chi_{0} H$ derived from our best fit. The curve passing through the data points is the fit to the theory of reference [3], expression ( $\left.1^{\prime}\right)$.

The set of parameters that gives the best fit to the data are the following: $\Lambda^{2}=$ $0.63 \mu_{\mathrm{B}}, M_{\mathrm{s}}=6.52 \mu_{\mathrm{B}}, H_{\mathrm{c}} \approx 0, H_{\mathrm{co}}=2 A / M_{\mathrm{s}} R_{\mathrm{a}}^{2}=224 \mathrm{kOe}$ and $\chi_{0}=8.2 \times 10^{-4} \mu_{\mathrm{B}} / \mathrm{kOe}$. Interestingly the values of $M_{s}, H_{c}$ and especially $\chi_{0}$ are very close to what was a priori expected (see above). Furthermore the obtained parameters are unique because the error bars on the value of $H_{\text {co }}$ (which determines the other parameters) are very small (less than $1 \mathrm{kOe})$. This first verification of the expression (1') shows that :

i) although the cross-over between the $1 / \sqrt{H+H_{c}}$, and the $1 / H^{2}$ regimes occurs at the 
field $H \approx H_{\text {co }}$ which is larger than our maximum measuring field ( $\left.150 \mathrm{kOe}\right)$, the parameter $H_{\text {co }}$ can accurately be determined ;

ii) the correlation volume of anisotropy directions $\Omega=\int 4 \pi r^{2} C(r) \mathrm{d} r$, can be evaluated.

We find $\Omega=11.5 a^{3}$. Note that we tried to change $C(r)=\exp \left(-r / R_{\mathrm{a}}\right)$ into $C(r)=$ $\cosh ^{-2}\left(r / R_{\mathrm{a}}\right)$. This does not modify our fits.

This detailed analysis has been completed by the study of the other samples $(x>0.1)$ which satisfy Chudnovsky's assumption $D \ll J$ less and less when $x$ increases [5]. The obtained fitting parameters are given in tables I and II.

Table I. - Parameters obtained for the series a-Dy $\mathrm{Gd}_{1-x} \mathrm{Ni}$ from the plots described in the text. The Pauli susceptibility $\chi_{0}$ has been estimated as described in the text (the error bars never exceed $10^{-4} \mu_{\mathrm{B}} / \mathrm{kOe}$ ).

\begin{tabular}{|c|c|c|c|c|c|}
\hline$x$ & $B\left(\mu_{\mathrm{B}} \mathrm{kOe}^{1 / 2}\right)$ & $M_{\mathrm{s}}\left(\mu_{\mathrm{B}}\right)$ & $H_{\mathrm{c}}(\mathrm{kOe})$ & $H_{1}^{*}(\mathrm{kOe})$ & $\chi_{0}\left(\mu_{\mathrm{B}} / \mathrm{kOe}\right)$ \\
\hline 1 & 4.9 & 5.80 & 2.81 & 17 & $8 \times 10^{-4}$ \\
\hline 0.75 & 4.6 & 6.45 & 2.69 & 13 & $8 \times 10^{-4}$ \\
\hline 0.5 & 2.9 & 6.49 & 1.84 & 22 & $8 \times 10^{-4}$ \\
\hline 0.25 & 1.3 & 6.39 & 0.92 & 10 & $8 \times 10^{-4}$ \\
\hline 0.1 & 0.46 & 6.55 & 0 & 19 & $8 \times 10^{-4}$ \\
\hline
\end{tabular}

Table II. - Evolution across the series of the paramagnetic temperature $\theta_{p}$, the local anisotropy to exchange ratio $D / J$, the correlation volume of anisotropy directions $\Omega / a^{3}$ and the corresponding correlation length (with the assumption of a $C(r)$ such as $\Omega \approx R_{a}^{3}$ ). The ferromagnetic correlation length $R_{f}$ deduced from $J / D$ and $R_{a}$ is also given. ${ }^{*}$ This value, rather small, is difficult to determine. It is somewhat different from the one of reference [7] in which we used only the HPZ model.

\begin{tabular}{|c|c|c|c|c|c|c|}
\hline$x$ & $\theta_{\mathrm{p}}(\mathrm{K})$ & $D / J$ & $\Omega / a^{3}$ & $R_{\mathrm{a}} / a$ & $R_{\mathrm{f}} / a$ & $R_{\mathrm{f}}(\AA)$ \\
\hline 1 & 14 & 0.95 & 14.7 & 2.4 & 3.7 & 9.2 \\
\hline 0.75 & 25 & 0.74 & 16.2 & 2.5 & 6.2 & 15.6 \\
\hline 0.5 & 38.5 & 0.55 & 14.7 & 2.4 & 11.3 & 28.3 \\
\hline 0.25 & 51.5 & 0.32 & 16.9 & 2.6 & 32.5 & 81.3 \\
\hline 0.1 & 58.6 & $0.10^{*}$ & 14.5 & 2.4 & 340.0 & 848.0 \\
\hline
\end{tabular}


The recent literature shows that the magnetization curves of some soft amorphous materials have been fitted to the $1 / \sqrt{H}[8-13]$ law and, in one case, to $\Delta M \sim 1 / H^{2}[13]$ above the $1 / \sqrt{H}$ regime. In this last case, a third regime is observed above the $1 / H^{2}$ one. We believe this regime to be due to the Pauli susceptibility contribution. Furthermore our evaluation of the correlation length of random anisotropy directions in this $\mathrm{Fe} B$-based alloy led to the enormous value $R_{\mathrm{a}} \approx 2 \times 10^{2}-3 \times 10^{2} \AA$. If this value were true this would indicate that these alloys would either be polycrystals and not amorphous (which is contradicted by the Xray analysis [13]) or would present correlations of anisotropy directions over a lengthscale much larger than the short range scale positional order of amorphous alloys, and this is an extremely interesting possibility to be investigated in more detail.

\section{Approach to saturation in nanoparticles of $\mathrm{Fe}$ epitaxed in an $\mathrm{Al}_{2} \mathrm{O}_{3}$ matrix.}

The experiments have been performed in a conventional magnetometer providing a magnetic field up to $7 \mathrm{~T}$, in the temperature range 1.5 to $300 \mathrm{~K}$. The samples are nanocomposite particles of $\mathrm{Fe}$ epitaxed in a matrix of $\mathrm{Al}_{2} \mathrm{O}_{3}$. We have studied 5 samples with different $\mathrm{Fe}$ concentrations $(1.0,2,5,10$ and $15 \mathrm{wt} \%)$. We shall only describe here our results for a single composition $x=10 \%$. The results obtained for the other compositions [6] are very similar to and coherent with those obtained for $x=10 \%$.

Contrary to the case of amorphous alloys in which we have interpreted our experiments by a zero-Kelvin model, here we have fitted our $M(H)$ curves to a model in which the temperature plays a major role. The reason for that is the weakness of interparticleinteractions in comparison with the temperature and also with the applied magnetic fields. Interparticle correlations can be neglected whereas in Chudnovsky's model of correlated spinglasses (3) this is obviously not the case. In non-interacting nanoparticles the blocking temperature $T_{\mathrm{n}}^{\mathrm{B}}$ above which each particle $n$ becomes superparamagnetic can be very small (or very large) for a given set of size-distributed particles and therefore even if the measuring temperature is small the $M(H)$ curves will be very sensitive to the temperature making it difficult to use a model at $T=0 \mathrm{~K}$ (even if the analysis is restricted to classical mechanics).

In our model for NISP, we start from the expression

$$
M(H, T)=\int_{s_{\mathrm{m}}}^{s_{\mathrm{M}}} \mathrm{d} s n(s) m(s) \mathcal{L}\left(\frac{m(s) H}{k T}\right)
$$

of the total magnetization where $n(s)$ is the particle size $(s)$ distribution, $m(s)$ the particle magnetization and $\mathcal{L}(x)$ a Langevin function.

In analogy with the fractal cluster model [14] we have assumed the following functional dependences $n(s) \sim s^{-a}$ and $m(b) \sim m^{b}$ where $a$ and $b$ are positive exponents. The low and large size cut-off $s_{\mathrm{m}}$ and $s_{\mathrm{M}}$ have been evaluated for two different limits.

LOW FIELD LIMIT. $-M_{0} H \ll K$ where $K$ is an effective anisotropy energy and $M_{0}$ a magnetization per volume unit. The activation energy $E \approx\left(K-M_{0} H\right)$. $s$ will be dominated by $K$ and in a rough approximation $E \approx K s_{M} \approx k T \operatorname{Ln}\left(t / \tau_{0}\right)$. In this regime, the sizes of the particles contributing to the value of the magnetization are in between a «topological » lower size cut-off $s_{\mathrm{m}}$ and a «thermal $\gg$ upper size cut-off $s_{\mathrm{M}} \approx(k T / K) \mathrm{Ln}\left(t / t_{0}\right)$. For quasi-static experiments $\operatorname{Ln}\left(t / \tau_{0}\right) \approx 25$.

LARGE FIELD LIMIT. $-M_{0} H \gg K$. This approximation leads to the lower size cut-off $s_{\mathrm{m}} \approx\left(k T / M_{0} H\right) \operatorname{Ln}\left(t / \tau_{0}\right) \approx 25 k T / M_{0} H$ and to the upper one $s_{\mathrm{M}}=$ constant = largest 
particle of the distribution (topological). Insertıng these cut-off in equation (1) we immediately get the following scaling forms :

Low field regime.

$$
M_{\mathrm{B}}(H, T)=M_{0}\left(\frac{T}{H}\right)^{\frac{-a+b+1}{b}} f\left(\frac{H}{T^{1-b}}\right) .
$$

Large field regime.

$$
M_{\mathrm{L}}(H, T)=M(\infty, 0)-M_{1}\left(\frac{T}{H}\right)^{\frac{-a+b+1}{b}} g\left(\frac{H}{T}\right) .
$$

$M_{0}$ and $M_{1}$ are constant magnetizations and $M(\infty, 0)$ the saturation magnetization.

Our Fe particles show coercive fields smaller than $500 \mathrm{Oe}$ and dipolar interaction fields of less than $1 \mathrm{kOe}$, the scaling model given above can be used for the interpretation of the $M(H)$ curve of these samples provided the applied field is larger than $1 \mathrm{kOe}$.

The magnetization curves of figure 3 have been reploted according to the scaling expressions (2) and (3), for many different values of the exponents $c=(-a+b+1) / b$ and $b$. Excellent scaling plots to these expressions have been obtained for the set of parameters $a=1.32 \pm 0.03$ and $b=0.35 \pm 0.02$ figures $4 \mathrm{a}$ and $4 \mathrm{~b}$. Beyond these values data points begin to scatter all over the plane for both low and large fields limits. Using these exponents we have computed the $M(H)$ curves between 1.5 and $300 \mathrm{~K}$ [6] (polynomial expansions have been considered for the functions $f$ and $g$ ).

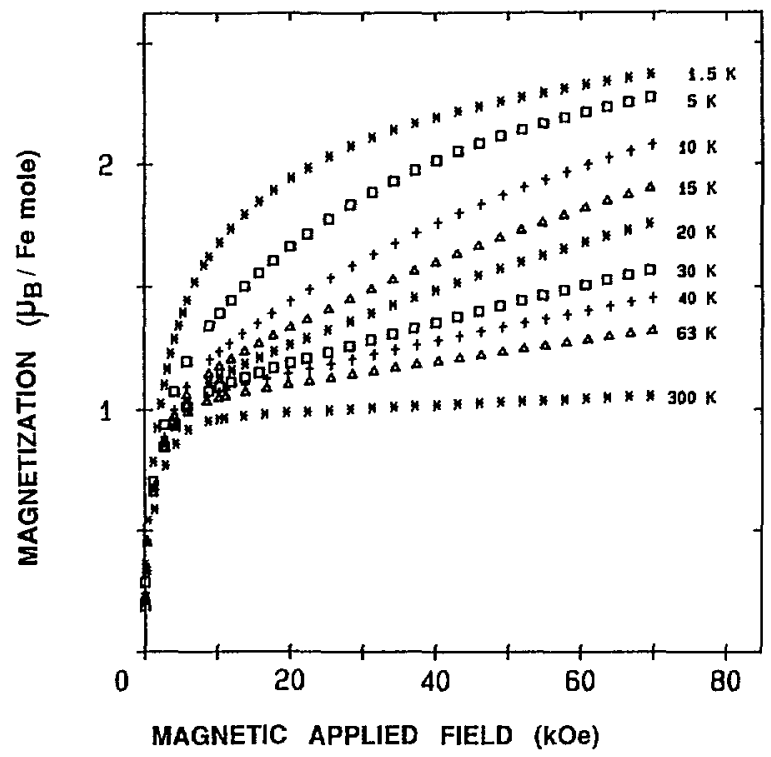

Fig. 3. - Raw magnetization curves for the sample $x=0,10$.

We shall end this paragraph by giving the physical meaning of the exponents $a$ and $b$. The size distribution $n(s)$ can be normalized to the Fe concentration $x, n(s)=n_{0} \cdot x\left(s / s_{0}\right)^{-a}$. This form compares very well with histograms deduced from TEM for each value of $x$. In the preceding formula $s_{0}$ represents the interatomic volume of the $\mathrm{Fe}$ particles and $n_{0}$ is a constant. 


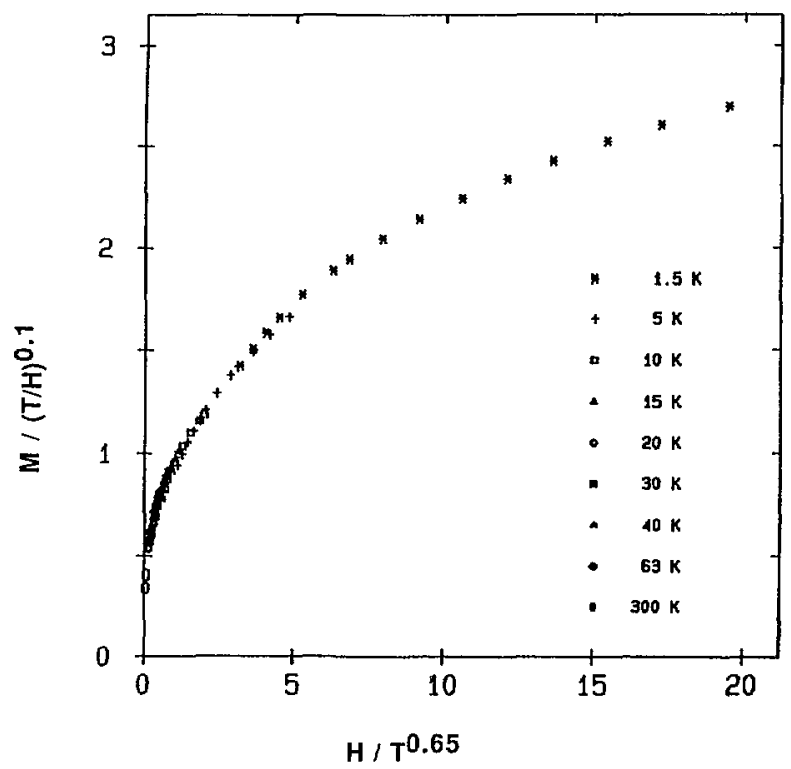

a)

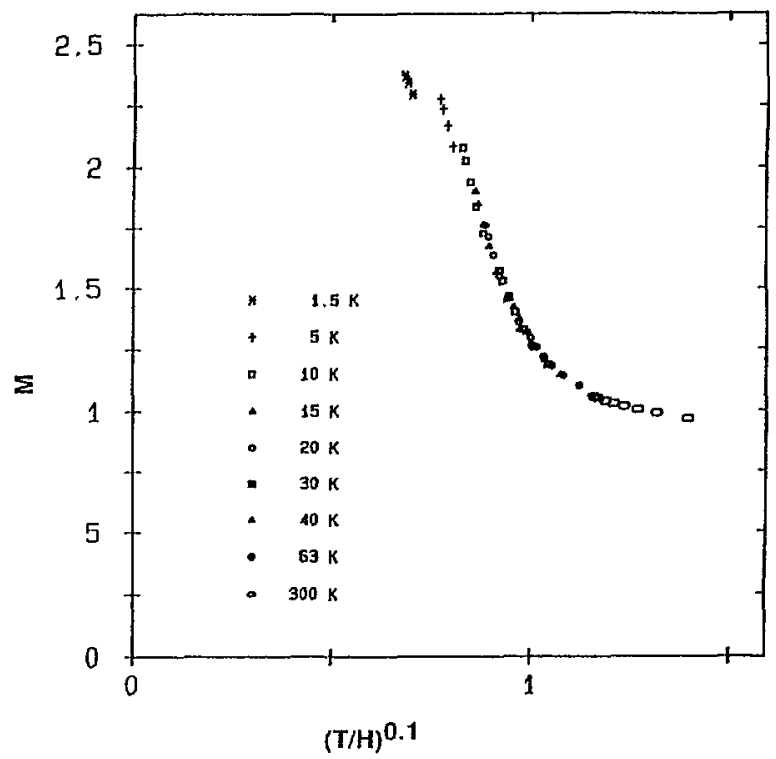

b)

Fig. 4. - Scaling plots of our data points for the low field regime (a) and the large field regime (b).

Note that the agreement is obtained only after a normalization at $s=s_{\mathrm{m}}$. Concerning the exponent $b$ we remark that its value, smaller than 1 , indicates that the moment per Fe atom, $m=m(s) / s=m_{0}\left(s / s_{0}\right)^{b-1}$, in a particle of size $s$ increases when the particle becomes smaller $\left(m_{0}\right.$ and $s / s_{0}$ represent respectively the theoretical moment of $\mathrm{Fe}^{0}$ and the number of $\mathrm{Fe}$ atoms in a particle of size $s$ ). We have interpreted this observation by assuming that each particle of $\mathrm{Fe}^{0}$ is surrounded by a shell of one or two interatomic distances in which iron is ionic $(2+$ and 
or 3+). As a matter of fact (i) Mössbauer experiments often show the existence of $3+$ iron in Fe fine particles [15]. (ii) In our particles Mössbauer experiments have detected a small amount of trivalent ions [16] and (iii) if the reduction of the precursor alloys $\mathrm{Al}_{2-x} \mathrm{Fe}_{x} \mathrm{O}_{3}$ is performed at a lower temperature each particle is surrounded by a well defined shell of Al-Fe oxide [17]. We might therefore assume that even in good epitaxed $\mathrm{Fe} / \mathrm{Al}_{2} \mathrm{O}_{3}$ interface there are still some divalent and/or trivalent iron ions reminiscent of this mixed oxide (essentially $\mathrm{Fe}$ ions with a $\mathrm{Fe}_{x} \mathrm{Al}_{2}-\mathrm{O}_{3}$ type of short range order). Within this picture the magnetization per $\mathrm{Fe}$ atom can be written (for spherical particles) :

$$
m=3 m_{1}\left(\frac{e}{R}\right)+m_{0}\left(1-\frac{e}{R}\right)^{3}
$$

where $e$ represents the thickness of the interface, $m_{0} \approx 2.2 \mu_{\mathrm{B}}$ and $m_{1} \approx 4-5 \mu_{\mathrm{B}}$ metallic and ionic Fe moments. Since $R=(3 s / 4 \pi)^{1 / 3}$, the preceding expression (4) can be compared to $m(s) / s=m_{0}\left(s / s_{0}\right)^{b-1}$. The very similar shapes of these two curves allow us to determine the thickness of the $\mathrm{Fe} / \mathrm{Al}_{2} \mathrm{O}_{3}$ interface $: e \approx 7 \AA$. We found the same value for each sample. It corresponds exactly to 2 interatomic distances!

\section{Conclusion.}

We have taken two examples of systems where the ferromagnetic correlation length does not diverge due to disorder-induced lengthscale cut-off. We have shown how high-field magnetization curves are sensitive to these cut-off and how far one can go in the analysis of these curves. In the case of amorphous alloys the $1 / H^{2}$ mean-field regime is preceded by a non mean field $1 / \sqrt{H}$ limit. This last regime vanishes in long range ferromagnets (i.e. when the uniform anisotropy field $H_{\mathrm{c}}$ becomes equal to a sizable fraction of the random anisotropy field $H_{\mathrm{r}}$ ). A detailed interpretation of the $M(H)$ curves at $T=0 \mathrm{~K}$ allows the determination of all the important parameters including the lengthscales of the ferromagnetic correlations $\left(R_{\mathrm{f}}\right)$ and of anisotropy direction correlations $\left(R_{\mathrm{a}}\right)$.

In the case of ferromagnetic nono-particles we can understand in detail the magnetization curves at any temperature between 2 and $300 \mathrm{~K}$ and any field above $1 \mathrm{kOe}$ up to $7 \mathrm{~T}$. In particular we show that these magnetization curves are very sensitive to a two-interatomic distance interface taking place between the epitaxed $\mathrm{Fe}$ particles and the $\mathrm{Al}_{2} \mathrm{O}_{3}$ matrix. Together with the thickness of the interface we have determined the size distribution of the particles for different $\mathrm{Fe}$ concentrations.

Let us end this conclusion by noting that our scaling forms for nanoparticles (Eqs. (2) and (3)), although showing in general analycity-breaking, could be for certain values of the exponents similar to Chudnovsky's result for amorphous magnets with two limiting regimes $1 / \sqrt{H}$ and $1 / H$. However, as mentioned in the introduction, amorphous alloys and noninteracting nanoparticles show important differences : in amorphous alloys magnetic correlations are important and classical spin-waves cannot be excited at very low temperatures. In such a case a zero-Kelvin model is possible. In non-interacting nanoparticles (i.e. for $H>1 \mathrm{kOe}$ in our case) magnetic fluctuations are localized on each particle and they persist even at very low temperatures for the smaller particles (superparamagnetism). The effect of temperature cannot be neglected in such a case. Furthermore quantum corrections should become essential at sufficiently low temperature (tunneling between two particle magnetization states). In low fields, when dipolar interactions are relevant, thermal fluctuations of the moment of individual particles should slow down in time and expand in space leading to real magnetic excitations. These excitations should be similar to those in spin-glasses or of random 
anisotropy systems. Small Angle Scattering experiments on these systems are scheduled. Finally let us mention that we have generalized our scaling model to the dynamical case, and ac susceptibility experiments will now allow us to test this model at different frequencies.

\section{References}

[1] IMrY Y. and MA S., Phys. Rev. Lett. 35 (1975) 1399.

[2] Chudnovsky E. M., Saslow W. M. and Serota R. A., Phys. Rev. 33 (1986) 251.

[3] Chudnovsky E. M., J. Magn. Magn. Mater. 79 (1989) 127.

[4] For a review see Chudnovsky E. M., J. Appl. Phys. 64 (1988) 5770.

[5] Filippi J., Amaral V. S. and Barbara B., Phys. Rev. Rap. Commun. (to appear).

[6] Marchand A., Barbara B., Mollard P., DevauX X. and Rousset A., Submitted to Europhys. Lett.

[7] Filippi J., Dieny B. and Barbara B., Solid State Commun. 53 (1985) 523.

[8] Sellmeyer D. J. and Nafis S., J. Appl. Phys. 57 (1985) 3584.

[9] Lee K. M., O'Shea M. J. and Sellmeyer D. J., APS March meeting 1988, New Orleans.

[10] O'Shea M. J., Lee K. M. and Fert A., J. Appl. Phys. 67 (1990) 9, 5769.

[11] Park M. J., Bhagat S. M., Manheimer M. A. and Moorjani K., Phys. Rev. B 33 (1986) 2070.

[12] Gehring P. M., Salamon M. B., Del Moral A. and Arnaudas J. I., Phys. Rev. B 41 (1990) 9134.

[13] Tejada J., Martinez B., Labarta A., Grossinger R., Sassik H., Vazquez M. and Hernando A., Phys. Rev. B 42 (1990) 898.

[14] Malozemoff A. P. and Barbara B., J. Appl. Phys. 57 (1985) 1, 3410.

[15] Fiorani D., Tholence J. and Dormann J. L., J. Phys. C : Solid State Phys. 19 (1986) 5495-5507.

[16] Sanchez J., Marchand A., Barbara B., Mollard P., DevauX X. and Rousset A., to be published.

[17] Marchand A., Barbara B., Devaux X., Mollard P. and Rousset A., to be published. 